\section{Influência de hábitos antrópicos na dispersão de Triatoma pseudomaculata Corrêa \& Espínola, 1964, através de Mimosa tenuiflora (Willdenow) (Mimosaceae) no Estado do Ceará, Brasil}

\author{
Influence of anthropic habits in the dispersion \\ of Triatoma pseudomaculata Corrêa \& Espínola, \\ 1964 through Mimosa tenuiflora (Willdenow) \\ (Mimosaceae) in the State of Ceará, Brazil
}

Simone Patrícia Carneiro Freitas 1 Assilon Lindoval Carneiro Freitas 2 Severino do Monte Prazeres 3 Teresa Cristina Monte Gonçalves 1
1 Núcleo de Morfologia e Ultraestrutura de Vetores,

Laboratório de Transmissores de Leishmanioses,

Departamento

de Entomologia,

Instituto Oswaldo Cruz,

Fundação Oswaldo Cruz,

Rio de Janeiro, Brasil.

2 20a Célula Regional

da Saúde, Crato, Brasil.

3 Departamento de Botânica,

Universidade Federal de

Pernambuco, Recife, Brasil.

Correspondência

Simone Patrícia

Carneiro Freitas

Núcleo de Morfologia

e Ultraestrutura de Vetores,

Laboratório de Transmissores

de Leishmanioses,

Departamento de

Entomologia,

Instituto Oswaldo Cruz,

Fundação Oswaldo Cruz.

Av. Brasil 4365,

Rio de Janeiro, $R$

21045-900, Brasil.

sfreitas@ioc.fiocruz.br

\begin{abstract}
Triatoma pseudomaculata was captured in the phloem of the black acacia bush (Mimosa tenuiflora) in seven municipalities in the State of Ceará, Brazil. This bush is widespread in the caatinga (scrub forest) and is used extensively by the rural population for kindling. Peridomiciliary presence of triatomines in the Cariri region may be associated with the use of infested M. tenuiflora wood by the population, thus facilitating the triatomines' mobilization and dispersion
\end{abstract}

Triatoma; Mimosa; Vectors Ecology
Triatoma pseudomaculata Corrêa \& Espínola 1964, espécie peridomiciliar, com distribuição geográfica nas regiões semi-áridas e de clima quente, é encontrada próxima à espécie Triatoma brasiliensis Neiva 1911, e esta geralmente ocupa habitats domésticos. Estudos recentes mostram que o peridomicílio é o principal fator de infestação do domicílio em áreas ocupadas por T. brasiliensis e T. pseudomaculata, respondendo por cerca de $80 \%$ dos casos; porém, mantê-lo com baixa infestação pode apresentar uma barreira à entrada desses triatomíneos no interior das casas 1,2 .

As caatingas predominam como fitofisionomia natural na região semi-árida do Nordeste Brasileiro, ocupando uma área de aproximadamente 800 mil km² 3. O Estado do Ceará, com uma cobertura vegetal predominante de caatingas, possui uma vasta área ruralizada, com habitações humanas e hábitos cotidianos que propiciam abrigo e mobilização de espécies de triatomíneos de importância na saúde pública. De acordo com Dias-Lima \& Sherlock 4, a destruição e/ou transformação dos ecótopos naturais podem causar a diminuição ou até mesmo o desaparecimento das fontes alimentares dos triatomíneos, resultando em pressão de invasão de domicílios e peridomicílios por esses vetores, não somente na busca de complementação ou dieta completa com sangue humano, e/ou de animais domésticos e/ou sinantrópicos, mas também na busca de novos abrigos. 
Assim, esta condição factual, transforma-se num perigo expondo a população aos riscos da doença de Chagas.

Dentre as espécies de plantas encontradas na caatinga, Mimosa tenuiflora (Willdenow), vulgarmente conhecida como "jurema-preta", apresenta predomínio densitário e uma alta taxa de cobertura geográfica, distribuindo-se no Brasil no Estado do Ceará, Rio Grande do Norte, Piauí, Bahia e fronteira com Minas Gerais 5. As populações humanas nas áreas rurais usam caules e galhos desta vegetação para proteção e isolamento dos animais de criação, e como fonte energética para cozinhar. Neste procedimento mantém a planta acumulada no peridomicílio.

Capturas de triatomíneos foram realizadas em ecótopos silvestres nas áreas de cobertura de M.tenuiflora, de sete municípios: Antonina do Norte, Assaré, Campos Sales, Potengi, Sali- tre, Tarrafas e Várzea Alegre, localizados na Região do Cariri, no sul do Estado do Ceará (Figura 1). As capturas foram feitas utilizando-se armadilhas 6 que continham pintos como isca, sendo colocadas no tronco das árvores, em buracos de rochas e possíveis abrigos de animais silvestres, do período vespertino ao matutino, perfazendo um total de $12 \mathrm{~h}$ de exposição de isca. Paralelamente também foram realizadas buscas de triatomíneos, por um período de quatro horas, entre o período vespertino e matutino, envolvendo cerca de cinco pessoas que descascavam as árvores de jurema-preta predominantes na área.

Os espécimes capturados foram identificados como T. pseudomaculata. Do total de 84 ninfas capturadas, quatro foram com armadilha: uma ninfa de 4o estádio em Antonina e uma ninfa de 5o estádio em Campos Sales, Potengi e Tarrafas. Descascando-se as juremas-

Figura 1

Mapa da área estudada na região do Cariri, Estado do Ceará, Brasil. Em destaque na área estudada, os municípios de captura de Triatoma pseudomaculata. Sede da 20ạ Célula Regional da Saúde, Crato, Ceará, Brasil.

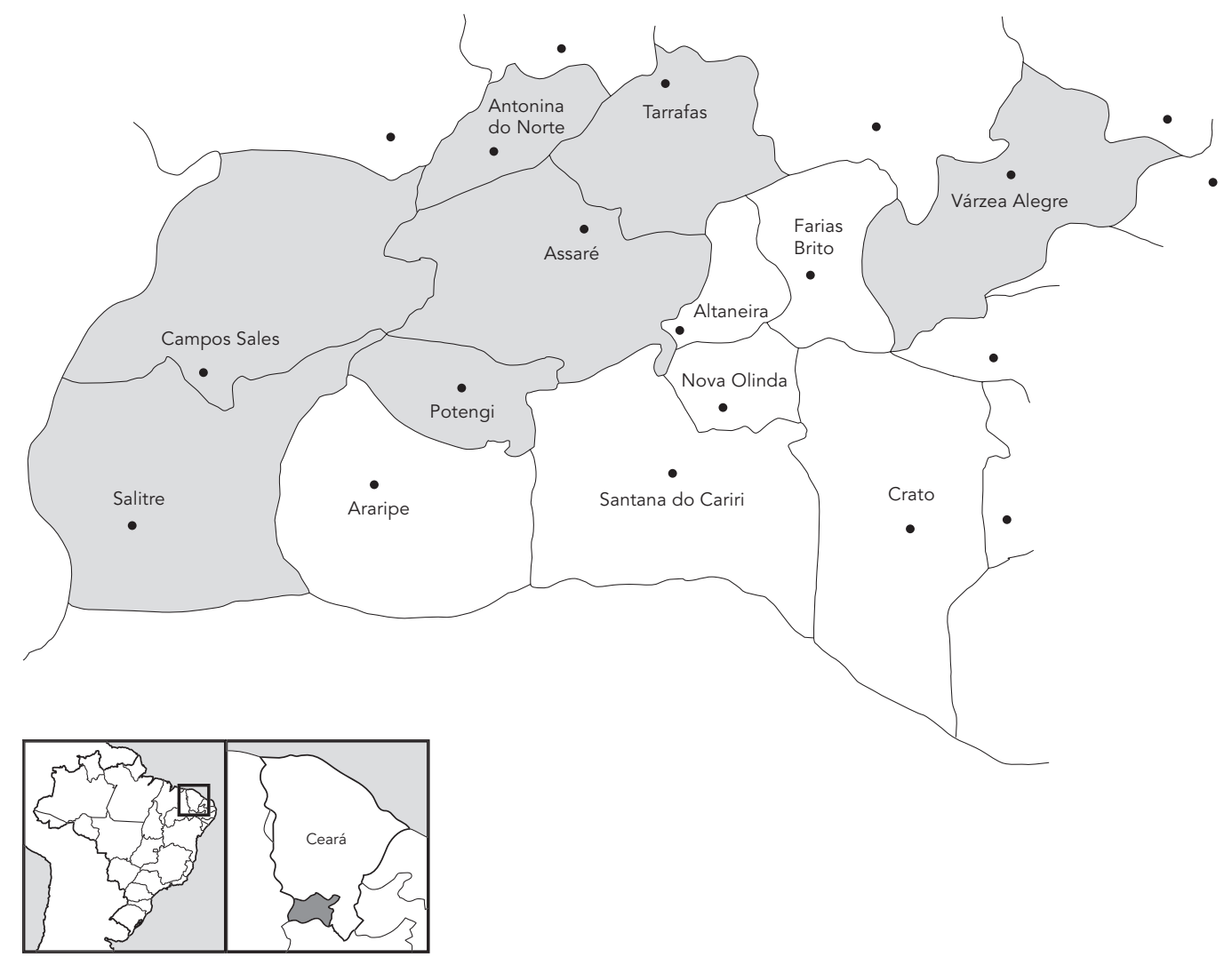


pretas, foi capturado um número maior de espécimes: sete ninfas (duas de 2 o e 30 estádios, respectivamente, e três do 4 o estádio) em Antonina; dez ninfas (uma de lo estádio, duas de 2o, 3o e 5o estádios, respectivamente, e três do 4o estádio) em Assaré; três ninfas (uma do 2o, 3o e 5o estádios, respectivamente) em Campos Sales; um adulto e 24 ninfas (três do 1o estádio, cinco do 2oㅡ, 3o e 4o estádios, respectivamente, e seis do 5o estádio) em Potengi; 30 ninfas (cin-

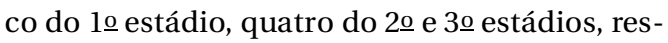

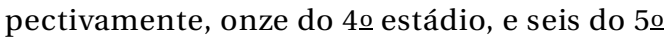
estádio) em Salitre; duas ninfas de 5o estádio em Tarrafas e quatro ninfas (uma de 4o estádio e três de 5o estádio) em Várzea Alegre (Tabela 1). Além disso, foram vistos ninhos de aves, e alguns roedores habitando nas entrecascas.

A presença de vários estados homogenéticos, incluindo adulto, indica potencial de colonização e fundação por parte do fluxo ou batelada populacional. Naturalmente que o sucesso de fundação (efeito fundador) vai depender da pressão seletiva do meio, enquanto o estoque genético invasor, possibilitará a plasticidade fenótica de adaptabilidade às novas circunstâncias.

A domiciliação triatomínea é um comportamento populacional relativamente associado ao homem, e sua dispersão deve estar sujeita à atuação deste no meio natural 7. Uma vez que os triatomíneos são estritamente hematófagos, ficam dependentes de locais de abrigo e criação de fonte alimentar vertebrada. Esses dados nos levam a associar a presença de triatomíneos no peridomicílio, na região do Cariri (Freitas, dados não publicados), já que as populações humanas levam para casa, e em torno da mesma, a madeira de $M$. tenuiflora, do ambiente silvestre, que poderão conter triatomíneos na forma jovem, facilitando conseqüentemente sua mobilidade e dispersão. $\mathrm{O}$ acúmulo da madeira próximo das casas serve de abrigo para animais sinantrópicos, como gambá e roedores, possibilitando a presença constante do triatomíneo no local. A persistência dos focos naturais destas populações mantém, portanto, o processo de infestação nos anexos peridomiciliares 1,8 .
Tabela 1

Número de espécimes de Triatoma pseudomaculata capturados em municípios do Estado do Ceará, Brasil.

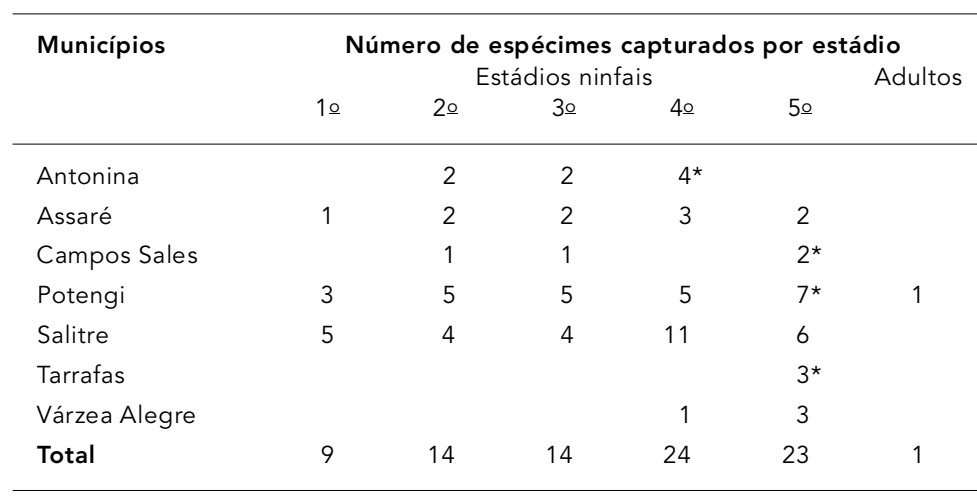

* Um espécime capturado em armadilha.

Conforme já observado por Coimbra-Filho \& Câmara 9, a aridez que assola algumas áreas do Nordeste vem aumentando gradativamente, parte como resultante da ação humana mudando a paisagem, estabelecendo novos ecótopos e alterando balanço hídrico. A invasão de M.tenuiflora, espécie xerófila secundária, em ambientes anteriormente em homeostase, torna-se cada vez mais extensa nas áreas semiáridas. Somado a esta condição o corte indiscriminado da vegetação monoespecífica está provocando empobrecimento ambiental e desertificação fitofisiológica. Desta forma, atenção deve ser dada ao fato do uso da sua madeira pelas populações humanas na região do Cariri, uma vez que tal condição é facilitadora para a mobilização e dispersão dos triatomíneos do ambiente natural para o peridomicílio.

Ressalta-se a atenção que deve ser dada à presença de T. pseudomaculata, na área estudada, porque a taxa de infestação nos municípios estudados vem se mantendo alta, bem como a taxa de positividade para T. cruzi (dados não publicados). Tal fenômeno de sucessão ecológica deve ser levado em conta no planejamento estratégico de controle sanitário/epidemiológico. 


\section{Resumo}

Triatoma pseudomaculata foram capturados em entrecascas de Mimosa tenuiflora (jurema-preta) em ecótopo silvestre de sete municípios do Estado do Ceará. Esta planta apresenta alta taxa de cobertura geográfica nas áreas de caatingas, sendo freqüentemente utilizada pela população rural. A presença de triatomíneos no peridomicílio, na região do Cariri, pode estar associada ao uso pela população da madeira de $\mathrm{M}$. tenuiflora, quando trazida do ambiente silvestre, repleta de triatomíneos, facilitando conseqüentemente sua mobilização e dispersão.

Triatoma; Mimosa; Ecologia de Vetores

\section{Colaboradores}

S. P. C. Freitas participou do trabalho de campo, coleta de dados e redigiu o artigo. A. L. C. Freitas contribuiu no trabalho de campo. S. M. Prazeres foi responsável pela identificação e dados das plantas. T. C. M. Gonçalves colaborou na revisão do artigo.

\section{Agradecimentos}

À Profa. Jacenir Reis dos Santos-Mallet, do Departamento de Entomologia do Instituto Oswaldo Cruz, à Profa. Márcia Pires, do Departamento de Sistemática Botânica do Jardim Botânico do Rio de Janeiro pelas valiosas informações sobre Mimosa tenuiflora e aos Agentes de Endemias da 20a Célula Regional da Saúde, Crato, Ceará, que muito contribuíram para a realização deste trabalho.

\section{Referências}

1. Oliveira Filho AM, Melo MTV, Santos CE, Faria Filho OF, Carneiro FCF, Oliveira-Lima JW, et al. Tratamento focais e totais com inseticida de ação residual para o controle de Triatoma brasiliensis e Triatoma pseudomaculata no nordeste brasileiro. Cad Saúde Pública 2000; 16 Suppl 2:105-111.

2. Soares RPP, Evangelista LG, Laranja LS, Diotaiuti, L. Population dynamics and feeding behavior of Triatoma brasiliensis and Triatoma pseudomaculata, main vectors of Chagas disease in northeastern Brazil. Mem Inst Oswaldo Cruz 2000; 95:151-5.

3. Araújo LVC. Levantamento fitossociológico da reserva particular do patrimônio natural da Fazenda Tamanduá, Santa Teresinha - Paraíba. Patos: Instituto Brasileiro do Meio Ambiente e dos Recursos Naturais Renováveis; 2000.

4. Dias-Lima AG, Sherlock IA. Sylvatic vectors invading houses and the risk of emergence of cases of Chagas disease in Salvador, State of Bahia, Northeast Brazil. Mem Inst Oswaldo Cruz 2000; 95:611-3.

5. Barneby RC. Sensitivae censitae. A description of the genus Mimosa Linnaeus (Mimosaceae) in the New World. Mem N Y Bot Gard 1991; 65:1-835.
6. Noireau F, Abad-Franch F, Valente SAS, Dias-Lima A, Lopes CM, Cunha V, et al. Trapping Triatominae in sylvatic habitats. Mem Inst Oswaldo Cruz 2002; 97:61-3.

7. Forattini OP. Biogeografia, origem e distribuição da domiciliação de triatomíneos no Brasil. Rev Saúde Pública 1980; 14:265-99.

8. Forattini OP, Barata JMS, Santos JLF, Silveira AC. Hábitos alimentares, infecção natural e distribuição de triatomíneos domiciliados na Região Nordeste do Brasil. Rev Saúde Pública 1981; 15:113-64.

9. Coimbra Filho AF, Câmara IG. Os limites originais do bioma Mata Atlântica na região Nordeste do Brasil. s.l.: Fundação Brasileira para a Conservação da Natureza; 1996

Recebido em 9/Jul/2003

Versão final reapresentada em 8/Out/2003 Aprovado em 28/Out/2003 